\title{
Analysis of Risk Factors of Level V Lymphatic Metastasis for Papillary Thyroid Carcinoma with pN1b
}

\author{
Chuang Li, ${ }^{1,2}$ Zhao-zhong Meng, ${ }^{1}$ Jian-wu Qin $\mathbb{D}^{1},{ }^{1}$ and Xin-guang Qiu $\mathbb{D}^{2}$ \\ ${ }^{1}$ Department of Thyroid and Neck, The Affiliated Cancer Hospital of Zhengzhou University, Henan Cancer Hospital, \\ Zhengzhou 450003, China \\ ${ }^{2}$ Department of Thyroid Surgery, The First Affiliated Hospital, Zhengzhou University, Zhengzhou 450052, China
}

Correspondence should be addressed to Jian-wu Qin; qinjianwu2021@163.com and Xin-guang Qiu; qiuxinguang2021@163.com

Received 30 January 2021; Revised 4 June 2021; Accepted 10 August 2021; Published 19 August 2021

Academic Editor: Amir Radfar

Copyright (C) 2021 Chuang Li et al. This is an open access article distributed under the Creative Commons Attribution License, which permits unrestricted use, distribution, and reproduction in any medium, provided the original work is properly cited.

Objective. To explore the risk factors of level V lymphatic metastasis in papillary thyroid carcinoma (PTC) patients with pN1b. Methods. Patients were selected if they presented with a suspicious level III or IV lymph node metastasis and underwent surgery by hemi or total thyroidectomy with a lymph node dissection (levels III, IV, VI, and VII). For these patients, if frozen section showed a positive level III or IV node, then levels II and V nodes were resected. Univariate analysis was performed using the chi-square test for some factors, including age, sex, tumor location, multifocal lesions, tumor size, local invasion of primary focus, status of cervical lymphatic metastasis, TNM staging, tumor deposits (independent tumor nodules), and the metastasis to more than 5 central lymph nodes. Then, the factors with statistical significance indicated by the above univariate analysis underwent multivariate analysis. Results. Univariate analysis indicated that the level V lymphatic metastasis was significantly associated with simultaneous metastases to levels II, III, and IV, simultaneous metastases to levels III and IV, and tumor deposits (all $p<0.05$ ), but it was not significantly associated with age, sex, tumor location, multifocal lesions, tumor size, local invasion of primary focus, other cervical lymphatic metastasis, TNM staging, and the metastases to more than 5 central lymph nodes (all $p>0.05$ ). Multivariate analysis suggested that the simultaneous metastases to levels III and IV and tumor deposits were the risk factors of level V lymphatic metastasis. Conclusion. The simultaneous metastases to levels III and IV and tumor deposits are independent risk factors of level V lymphatic metastasis. The patients with pN1b PTC who have simultaneous metastases to levels III and IV or/ and tumor deposits may have the risk of level V lymph node metastasis.

\section{Introduction}

Papillary thyroid carcinoma (PTC), a common pathological type, accounts for approximately $85 \%$ of thyroid cancer [1]. In recent 10 years, PTC incidence has been increasing in the world. In PTC, cervical lymph node metastasis is common with a metastasis rate of as high as $40 \%-90 \%$ [2]. PTC metastasis often occurs in the ipsilateral lymph nodes of the primary lesion and is usually along the following lymphatic course: first, the central region (levels VI-VII), then jugular chain lymph nodes (levels II-IV), and finally the posterior cervical lymph nodes (level V), but skip metastasis may also occur [3]. Jugular chain lymph node metastasis affects local tumor recurrence and disease-free survival [4]. American Thyroid Association guidelines made in 2015 recommended lateral neck lymph node dissection for the patients with biopsy-confirmed jugular chain lymph node metastasis [5]. However, it is still controversial whether the patients with lateral neck lymph node metastasis require further level $\mathrm{V}$ lymph node dissection. Preoperative ultrasound and CT usually cannot detect level V lymph node metastasis in the patients with PTC. In this study, we analyzed the risk factors of level V lymphatic metastasis in the PTC patients with $\mathrm{pN} 1 \mathrm{~b}$ to provide a reference for making treatment decisions.

\section{Subjects and Methods}

All study methods were approved by the Ethics Committee of the Affiliated Cancer Hospital of Zhengzhou University. 
All the subjects enrolled into the study gave written informed consent to participate.

2.1. Subjects. The inclusion criteria were (1) over the age of 18 years, (2) PTC confirmed by preoperative pathology, (3) PTC with pN1b identified by intraoperative frozen pathology, and (4) complete clinical data. The exclusion criteria included (1) other types of thyroid cancer, (2) combined with other malignancies, (3) a history of surgery or radiotherapy on the head and neck, (4) recurrent PTC, and (5) incomplete clinical data.

From January 2013 to December 2016, a total of 649 PTC patients with suspicious lateral neck metastasis underwent hemi- or total thyroidectomy with node dissection (levels III, IV, VI, and VII). If his/her intraoperative frozen pathology displayed $\mathrm{pN} 1 \mathrm{~b}$, the patient further received ipsilateral levels II and V lymph node dissection.

2.2. Surgery and Main Outcome Measures. Patients underwent color ultrasound examinations for the thyroid and neck before operation. If the color ultrasound showed suspicious metastatic lymph nodes in the levels III or/and IV, such as increased lymph node combined with calcification or liquefaction, all the lymph nodes and fatty tissue in the levels III and IV were removed, and all lymph node dissection specimens in the levels III and IV underwent rapid frozen pathological examination. If the color ultrasound indicated suspicious metastatic lymph nodes on bilateral levels III or/and IV, bilateral levels III and IV lymph node dissections were performed. If intraoperative frozen pathology displayed $\mathrm{pN} 1 \mathrm{~b}$, the patient further received ipsilateral levels II and V lymph node dissection. In this study, the lymph node metastasis included micrometastasis $(<2 \mathrm{~mm})$ and macrometastasis. All patients enrolled in this study underwent at least radical thyroid resection combined with ipsilateral modified levels II-VII lymph node dissection. The items, including age, sex, tumor location, tumor-multifocal, tumor size, local invasion of primary focus, status of cervical lymphatic metastasis, TNM staging, tumor deposits (independent tumor nodules), and the metastases to more than 5 central lymph nodes were recorded. A lymph node was completely replaced by tumor tissues, which was regarded as tumor deposits. The tumor deposits were identified by $\mathrm{H}$. E pathology of lymph node dissection. Under a microscope, the tumor deposits only contained thyroid malignant tissue without these structures, such as lymph tubes, nerves, or blood vessels.

2.3. Statistical Analysis. Statistical analysis was performed using SPSS19.0 software. The chi-square test was used in the comparison of qualitative data. And then, the data with statistical significance indicated by the above chi-square test underwent multivariate analysis using the logistic regression test. Statistical significance was established at $p<0.05$.

\section{Results}

3.1. General Data. pN1b PTC was identified in 132 patients from the 649 PTC patients. Of the 132 patients, 47 were male and 85 female, with a mean age of 42 years (range 19-77). The 132 patients underwent at least radical thyroid resection combined with ipsilateral modified levels II-VII lymph node dissection. In the 132 patients, 50 received ipsilateral modified levels II-VII lymph node dissection, 40 bilateral modified levels II-VII lymph node dissection, and 42 ipsilateral modified levels II-VII lymph node dissection combined with contralateral selective lymph node dissection. The status of cervical lymphatic metastasis is given in Table 1 . In the 132 patients, level V lymph node metastasis was found in 14 patients $(10.61 \%, 14 / 132)$ (Table 1). The tumor deposits occurred in 19 patients $(14.39 \%, 19 / 132)$ (Table 2).

\subsection{Univariate Analysis of the Level V Lymphatic Metastasis-} Related Factors. Univariate analysis indicated that the level $\mathrm{V}$ lymphatic metastasis was significantly associated with simultaneous metastases to levels II, III, and IV, simultaneous metastases to levels III and IV, and tumor deposits (all $p<0.05$ ), but it was not significantly associated with age, sex, tumor location, tumor-multifocal, tumor size, local invasion of primary focus, other cervical lymphatic metastasis, TNM staging, and the metastases to more than 5 central lymph nodes (all $p>0.05$ ) (Table 2 ).

3.3. Multivariate Analysis of the Level V Lymphatic MetastasisRelated Factors. The factors with statistical significance indicated by the above univariate analysis underwent multivariate analysis using the logistic regression test. Multivariate analysis suggested that the simultaneous metastases to levels III and IV and tumor deposits were the independent risk factors of level $\mathrm{V}$ lymphatic metastasis (all $p<0.05)$ (Table 3).

\section{Discussion}

The level V lymphatic metastasis is strongly associated with postoperative local tumor recurrence and disease-free survival in PTC [4], but its incidence is relatively low. The level $\mathrm{V}$ lymph node dissection may cause accessory nerve and cervical plexus injuries, which may lead to shoulder dysfunction, as well as numbness and neuralgia in the cervical region $[6,7]$. At present, little research has been performed on level V lymphatic metastasis in the PTC patients with pN1b. Chen et al. [8] reported that in 106 patients with $\mathrm{cN} 0$, 29 received levels II-V lymph node dissection, and level V lymphatic metastasis was found in 2 patients with a metastasis rate of $6.9 \%(2 / 29)$. The 2 patients with level V lymphatic metastasis had simultaneous metastases to levels II, III, and IV, so they recommended level V lymph node dissection for the patients with simultaneous metastases to levels II, III, and IV. Retrospective studies displayed that the level $\mathrm{V}$ lymphatic metastasis rate was between $12.3 \%$ and $53 \%$ in the patients with PTC undergoing II-VI lymph node 
TABLe 1: Cervical lymph node metastases in different levels in papillary thyroid carcinoma.

\begin{tabular}{lcc}
\hline Levels & Lymph node metastases $(n)$ & Metastasis rate (\%) \\
\hline II & 59 & $44.7(59 / 132)$ \\
III & 123 & $93.18(123 / 132)$ \\
IV & 93 & $70.45(93 / 132)$ \\
V & 14 & $10.61(14 / 132)$ \\
II and III & 53 & $40.15(53 / 132)$ \\
II and IV & 49 & $37.12(49 / 132)$ \\
III and IV & 79 & $59.85(79 / 132)$ \\
II, III, and IV & 44 & $33.33(44 / 132)$ \\
\hline
\end{tabular}

dissection [9-13]. Terrell et al. [7] found that the level V lymphatic metastasis rate was $16 \%$ in the patients who were clinically diagnosed with the negative level V lymph node. Shim et al. [11] reported that the level V lymphatic metastasis rate was $18.2 \%(26 / 143)$ in 143 patients who had lateral neck lymph node metastasis and received surgery for the first time. Wang et al. [12] retrospectively observed 1037 PTC patients with $\mathrm{cN} 1 \mathrm{~b}$ and found that the level V lymphatic metastasis rate was $21.3 \%$ (221/1037). Yang et al. [13] retrospectively analyzed 220 patients with solitary PTC and found that the level V lymphatic metastasis rate was $12.3 \%$. There is also considerable debate about the risk factors of the level V lymphatic metastasis. At present, most researchers believe that multiregion lymphatic metastasis among levels II, III, and IV is associated with the level V lymphatic metastasis, and it is an independent risk factor of the level $\mathrm{V}$ lymphatic metastasis $[9,13]$. Kupferman et al. [10] reported that the level V lymphatic metastasis was significantly related to tumor-multifocal lesions and ipsilateral lateral neck lymph node metastasis. Shim et al. [11] and Wang et al. [12] found that there was an independent correlation between lymph node-extracapsular spread and the level V lymphatic metastasis. Wang et al. [12] also found that lymph node $\geq 2 \mathrm{~cm}$, simultaneous metastases to levels II, III, and IV, and unilateral central lymph node metastasis were the independent predictors of the level $\mathrm{V}$ lymphatic metastasis $(p<0.05)$. Yang et al. [13] believed that the ipsilateral level V lymphatic metastasis was closely related to the simultaneous metastases to levels II-IV, tumor size $>1 \mathrm{~cm}$, extrathyroid invasion, ipsilateral central lymphatic metastasis rate $\geq 50 \%$, contralateral central lymph node metastasis (CLNM), and bilateral central metastasis, and CLNM was an independent risk factor of the level V lymphatic metastasis.

In the 132 patients, the level $\mathrm{V}$ lymphatic metastasis rate was $10.61 \%(14 / 132)$ which was higher than $6.9 \%$ reported by Chen et al. [8], similar to $12.3 \%$ reported by Yang et al. [13] and slightly lower than the results reported in references [9-16]. The various level V lymphatic metastasis rates may be related to the different indications of level $\mathrm{V}$ lymph node dissection. In this study, the PTC patients were diagnosed with positive $\mathrm{pN} 1 \mathrm{~b}$ by levels III and IV lymph node dissection, so the PTC patients enrolled in this study contained some PTC patients with lateral cervical occult metastasis. Compared with the PTC patients with positive $\mathrm{cN}+$, the course of disease was relatively shorter and the level $\mathrm{V}$ lymphatic metastasis rate was lower in this study.
In this study, the level V lymphatic metastasis-related factors underwent univariate analysis and multivariate analysis, and results suggested that the simultaneous metastases to levels III and IV and tumor deposit were the independent risk factors of level $\mathrm{V}$ lymphatic metastasis (all $p<0.05$ ). PTC metastasis is usually along the following lymphatic course: first, the central region (levels VI and VII), then lateral neck lymph nodes (levels II-IV), and finally the posterior cervical lymph nodes (level V, especially $\mathrm{Vb}$ ). In this study, the simultaneous metastases to the levels III and IV are an independent risk factor of the level V lymphatic metastasis, which may be that the levels III and IV are closer to the level Vb in space as compared with other lymph nodes. In this study, it was not found that the simultaneous metastases to the levels II, III, and IV was significantly associated with the level V lymphatic metastasis, which may be that the sample size of patients with the simultaneous metastases to the levels II, III, and IV was small and the PTC patients with the level II lymphatic metastasis rarely have level V lymphatic metastasis. This remains to be further confirmed by largesample clinical studies.

Tumor deposits, a special histopathological feature, were first discovered by Gabriel et al. [17] in rectal cancer in 1935. The tumor deposits are an important risk factor of prognosis in colorectal cancer and gastric cancer. It is defined as an independent presence of tumor nodules without identifiable lymph nodes, lymphatic vessels, nerves, or blood vessels [18]. The tumor deposit formation is not completely clear. It may originate from direct dissemination of tumors, lymph node metastasis, vascular invasion, or nerve invasion, and with tumor progresses, the original lymph node structure is destroyed followed by tumor deposits formation [19]. In AJCC staging, N stage only includes N1a and N1b, without considering the degree of metastatic lymph node invasion [20]. However, the degree of metastatic lymph node invasion may be associated with the risk of PTC recurrence [21]. At present, there are few studies on the tumor deposits of thyroid cancer, but tumor deposit formation is often found in the cervical lymph node dissection specimens. In this study, tumor deposits were found in 19 samples (19/132, $14.39 \%)$. The tumor deposits are usually associated with tumor recurrence and distant metastasis. In this study, both univariate analysis and multivariate analysis indicated that tumor deposit formation was significantly correlated with the level V lymphatic metastasis and is an independent risk factor of level V lymphatic metastasis. This suggests that the pN1b PTC patients with tumor deposits easily have lymph node metastasis. The tumor deposits may be regarded as lymph node-extracapsular spread because they have similar biological behaviors [22]. Shim et al. [11] and Wang et al. [12] found that there was an independent correlation between lymph node-extracapsular spread and the level V lymphatic metastasis. If the tumor deposits are regarded as lymph node-extracapsular spread, our results are similar to the results of Shim et al. [11] and Wang et al. [12].

In summary, the patients with pN1b PTC who have simultaneous metastases to levels III and IV, or/and tumor deposit, may have the risk of level $\mathrm{V}$ lymph node metastasis. 
TABLE 2: Univariate analysis of the factors associated with the level V lymph node metastases.

\begin{tabular}{|c|c|c|c|c|}
\hline \multirow{2}{*}{ Factors } & \multirow{2}{*}{ Patients $(n)$} & \multicolumn{2}{|c|}{ Cervical lymphatic metastasis } & \multirow{2}{*}{$P$} \\
\hline & & No & Yes & \\
\hline \multicolumn{5}{|l|}{ Age } \\
\hline$<55$ & 114 & $101(88.6 \%)$ & $13(21.44 \%)$ & \multirow{2}{*}{0.691} \\
\hline$\geq 55$ & 18 & $17(94.44 \%)$ & $1(10.44 \%)$ & \\
\hline \multicolumn{5}{|l|}{ Sex } \\
\hline Female & 85 & $76(89.41 \%)$ & $9(19.91 \%)$ & \multirow{2}{*}{1} \\
\hline Male & 47 & $42(89.36 \%)$ & $5(20 \%)$ & \\
\hline \multicolumn{5}{|l|}{ Tumor location } \\
\hline Upper & 18 & $18(100 \%)$ & $0(0 \%)$ & \multirow{5}{*}{0.1} \\
\hline Middle & 66 & $61(92.42 \%)$ & $5(14.24 \%)$ & \\
\hline Lower & 6 & $5(83.33 \%)$ & $1(31.33 \%)$ & \\
\hline Multifocal PTC & 34 & $28(82.35 \%)$ & $6(33.18 \%)$ & \\
\hline Whole lobe & 8 & $6(75 \%)$ & $2(47 \%)$ & \\
\hline \multicolumn{5}{|l|}{ Multifocal PTC } \\
\hline No & 83 & $75(90.36 \%)$ & $8(18.12 \%)$ & \multirow{2}{*}{0.638} \\
\hline Yes & 49 & $43(87.76 \%)$ & $6(23.02 \%)$ & \\
\hline \multicolumn{5}{|l|}{ Tumor size } \\
\hline$\leq 1 \mathrm{~cm}$ & 34 & $31(91.18 \%)$ & $3(16.59 \%)$ & \multirow{4}{*}{0.11} \\
\hline $1-2 \mathrm{~cm}$ & 53 & $50(94.34 \%)$ & $3(10.64 \%)$ & \\
\hline$>2 \mathrm{~cm}$ & 37 & $32(86.49 \%)$ & $5(25.41 \%)$ & \\
\hline$>4 \mathrm{~cm}$ & 8 & $5(62.5 \%)$ & $3(70.5 \%)$ & \\
\hline \multicolumn{5}{|l|}{ Local invasion } \\
\hline No & 50 & $45(90 \%)$ & $5(18.8 \%)$ & \multirow{3}{*}{0.105} \\
\hline Thyroid capsule & 34 & $33(97.06 \%)$ & $1(5.53 \%)$ & \\
\hline Outside capsule & 48 & $40(83.33 \%)$ & $8(31.33 \%)$ & \\
\hline \multicolumn{5}{|c|}{ Level II, III, and IV metastases } \\
\hline No & 88 & $83(94.32 \%)$ & $5(10.68 \%)$ & \multirow{2}{*}{$0.015^{*}$} \\
\hline Yes & 44 & $35(79.55 \%)$ & $9(38.45 \%)$ & \\
\hline Level II and III me & & & & \\
\hline No & 79 & $74(93.67 \%)$ & $5(11.9 \%)$ & \\
\hline Yes & 53 & $44(83.02 \%)$ & $9(31.92 \%)$ & 0.051 \\
\hline Level III and IV $\mathrm{m}$ & & & & \\
\hline No & 53 & $53(100 \%)$ & $1(3.55 \%)$ & \\
\hline Yes & 79 & $65(82.28 \%)$ & $13(30.94 \%)$ & $0.007^{*}$ \\
\hline$\overline{\text { Level II and IV me }}$ & & & & \\
\hline No & 83 & $75(90.36 \%)$ & $8(18.12 \%)$ & \\
\hline Yes & 49 & $43(87.76 \%)$ & $6(23.02 \%)$ & 0.638 \\
\hline TNM staging & & & & \\
\hline I & 107 & $99(92.52 \%)$ & $8(14.06 \%)$ & \\
\hline II & 13 & $9(69.23 \%)$ & $4(57.85 \%)$ & \\
\hline III & 8 & $7(87.5 \%)$ & $1(23.5 \%)$ & 0.12 \\
\hline IVB & 4 & $3(75 \%)$ & $1(47 \%)$ & \\
\hline Tumor deposits & & & & \\
\hline No & 113 & $105(92.92 \%)$ & $8(13.31 \%)$ & \\
\hline Yes & 19 & $13(68.42 \%)$ & $6(59.37 \%)$ & $0.006^{*}$ \\
\hline Metastases to over & nodes & & & \\
\hline No & 122 & $108(88.52 \%)$ & $14(21.57 \%)$ & \\
\hline Yes & 10 & $10(100 \%)$ & $0(0 \%)$ & 0.599 \\
\hline Level II metastases & & & & \\
\hline No & 73 & $66(90.41 \%)$ & $7(18.03 \%)$ & \\
\hline Yes & 59 & $52(88.14 \%)$ & $7(22.31 \%)$ & 0.673 \\
\hline Level III metastases & & & & \\
\hline No & 9 & $8(88.89 \%)$ & $1(20.89 \%)$ & \\
\hline Yes & 123 & $110(89.43 \%)$ & $13(19.87 \%)$ & 1 \\
\hline Level IV metastases & & & & \\
\hline No & 39 & $33(84.62 \%)$ & $6(28.92 \%)$ & \\
\hline Yes & 93 & $85(91.4 \%)$ & $8(16.17 \%)$ & 0.248 \\
\hline
\end{tabular}


TABLE 3: Multivariate analysis of independent risk factors of level V lymphatic metastasis.

\begin{tabular}{|c|c|c|c|c|c|c|}
\hline Factors & Regression coefficient & Statistic & Degree of freedom & $P$ & OR & $95 \% \mathrm{CI}$ \\
\hline \multicolumn{7}{|c|}{ Tumor deposits } \\
\hline No & Reference group & & & & & \\
\hline Yes & 2.977 & 11.713 & 1 & $0.001^{*}$ & 19.627 & $(3.568-107.955)$ \\
\hline \multicolumn{7}{|c|}{ Level III and IV metastases } \\
\hline No & Reference group & & & & & \\
\hline Yes & $3.272^{-1}$ & 6.797 & 1 & $0.009^{*}$ & 26.374 & $(2.253-308.712)$ \\
\hline \multicolumn{7}{|c|}{ Level II, III, and IV metastases } \\
\hline No & Reference group & & & & & \\
\hline Yes & 1.192 & 2.792 & 1 & 0.095 & 3.295 & $(0.814-13.344)$ \\
\hline
\end{tabular}

Notes: OR, odds ratio; CI, confidence interval. ${ }^{*} P<0.05$.

\section{Data Availability}

The data used to support the findings of this study are available from the corresponding author upon reasonable request.

\section{Conflicts of Interest}

The authors declare that they have no conflicts of interest.

\section{References}

[1] S. Zhan, D. Luo, W. Ge, B Zhang, and T Wang, "Clinicopathological predictors of occult lateral neck lymph node metastasis in papillary thyroid cancer: a meta-analysis," Head \& Neck, vol. 41, no. 4, pp. 2441-2449, 2019.

[2] C. I. Lundgren, P. Hall, P. W. Dickman, and J. Zedenius, "Clinically significant prognostic factors for differentiated thyroid carcinoma," Cancer, vol. 106, no. 3, pp. 524-531, 2006.

[3] C. Liu, C. Xiao, J. Chen et al., "Risk factor analysis for predicting cervical lymph node metastasis in papillary thyroid carcinoma: a study of 966 patients," BMC Cancer, vol. 19, no. 1 , p. $622,2019$.

[4] S. G. A. de Meer, M. Dauwan, B. de Keizer, G. D. Valk, I. H. M. Borel Rinkes, and M. R. Vriens, "Not the number but the location of lymph nodes matters for recurrence rate and disease-free survival in patients with differentiated thyroid cancer," World Journal of Surgery, vol. 36, no. 6, pp. 1262-1267, 2012.

[5] B. R. Haugen, E. K. Alexander, K. C. Bible et al., "2015 American thyroid association management guidelines for adult patients with thyroid nodules and differentiated thyroid cancer: the American thyroid association guidelines task force on thyroid nodules and differentiated thyroid cancer," Thyroid: Official Journal of the American Thyroid Association, vol. 26, no. 1, pp. 1-133, 2016.

[6] H. Inoue, K.-i. Nibu, M. Saito et al., "Quality of life after neck dissection," Archives of Otolaryngology-Head and Neck Surgery, vol. 132, no. 6, pp. 662-666, 2006.

[7] J. E. Terrell, D. E. Welsh, C. R. Bradford et al., "Pain, quality of life, and spinal accessory nerve status after neck dissection," The Laryngoscope, vol. 110, no. 4, pp. 620-626, 2000.

[8] R. Chen, T. Wei, M. Zhang et al., "Lateral neck lymph node metastasis in cN0 papillary thyroid carcinoma," Zhonghua er bi yan hou tou jing wai ke za zhi, vol. 47, no. 8, pp. 662-667, 2012.

[9] Y. C. Lim, E. C. Choi, Y.-H. Yoon, and B. S. Koo, "Occult lymph node metastases in neck level $\mathrm{V}$ in papillary thyroid carcinoma," Surgery, vol. 147, no. 2, pp. 241-245, 2010.

[10] M. E. Kupferman, Y. E. Weinstock, A. A. Santillan et al., "Predictors of level V metastasis in well-differentiated thyroid cancer," Head \& Neck, vol. 30, no. 11, pp. 1469-1474, 2008.
[11] M. J. Shim, J.-L. Roh, G. Gong et al., "Preoperative detection and predictors of level $\mathrm{V}$ lymph node metastasis in patients with papillary thyroid carcinoma," British Journal of Surgery, vol. 100, no. 4, pp. 497-503, 2013.

[12] Y. Wang, Q. Guan, and J. Xiang, "Nomogram for predicting level V lymph node metastases in papillary thyroid carcinoma with clinically lateral lymph node metastases: a large retrospective cohort study of 1037 patients from FDUSCC," Journal of Cancer, vol. 10, no. 3, pp. 772-778, 2019.

[13] J. Yang, Y. Gong, S. Yan, J. Zhu, Z. Li, and R. Gong, "Risk factors for level V lymph node metastases in solitary papillary thyroid carcinoma with clinically lateral lymph node metastases," Cancer Medicine, vol. 5, no. 8, pp. 2161-2168, 2016.

[14] H. S. Keum, Y. B. Ji, J. M. Kim et al., "Optimal surgical extent of lateral and central neck dissection for papillary thyroid carcinoma located in one lobe with clinical lateral lymph node metastasis," World Journal of Surgical Oncology, vol. 10, no. 1, p. 221, 2012.

[15] J.-W. Feng, A.-C. Qin, J. Ye, H. Pan, Y. Jiang, and Z. Qu, "Predictive factors for lateral lymph node metastasis and skip metastasis in papillary thyroid carcinoma," Endocrine $\mathrm{Pa}$ thology, vol. 31, no. 1, pp. 67-76, 2020.

[16] M. Javid, E. Graham, J. Malinowski et al., "Dissection of levels II through V is required for optimal outcomes in patients with lateral neck lymph node metastasis from papillary thyroid carcinoma," Journal of the American College of Surgeons, vol. 222 , no. 6, pp. 1066-1073, 2016

[17] W. B. Gabriel, C. Dukes, H. J. R. Bussey et al., "Lymphatic spread in cancer of the rectum," British Journal of Surgery, vol. 23, no. 90, pp. 395-413, 2010.

[18] M. B. Amin, F. L. Greene, S. B. Edge et al., "The Eighth Edition AJCC Cancer Staging Manual: continuing to build a bridge from a population-based to a more "personalized" approach to cancer staging," CA: A Cancer Journal for Clinicians, vol. 67, no. 2, pp. 93-99, 2017.

[19] I. D. Nagtegaal and P. Quirke, "Colorectal tumour deposits in the mesorectum and pericolon; a critical review," Histopathology, vol. 51, no. 2, pp. 141-149, 2007.

[20] G. Li, R. Li, L. Song et al., "Implications of extrathyroidal extension invading only the strap muscles in papillary thyroid carcinomas," Thyroid, vol. 30, no. 1, pp. 57-64, 2020.

[21] L. Jianyong, Z. Jinjing, L. Zhihui, W. Tao, G. Rixiang, and Z. Jingqiang, "A nomogram based on the characteristics of metastatic lymph nodes to predict papillary thyroid carcinoma recurrence," Thyroid, vol. 28, no. 3, pp. 301-310, 2018.

[22] I.-S. Lee, Y.-S. Park, M.-H. Ryu et al., "Impact of extranodal extension on prognosis in lymph node-positive gastric cancer," British Journal of Surgery, vol. 101, no. 12, pp. 1576-1584, 2014. 